\section{BRAZIULIAN JOURNAL}

OF MEDICAL AND BIOLOGICAL RESFARCH

www.bjournal.com.br
ISSN 0100-879X

Volume 43 (8) 698-811 August 2010

BIOMEDICAL SCIENCES

AND

CLINICAL INVESTIGATION

Braz J Med Biol Res, August 2010, Volume 43(8) 794-798

doi: 10.1590/S0100-879X2010007500067

Meningoencephalitis caused by a zygomycete fungus (Basidiobolus) associated with septic shock in an immunocompetent patient: 1-year follow-up after treatment

M. Auxiliadora-Martins, G.C. Alkmim-Teixeira, J. Machado-Viana, E.A. Nicolini, O.A. Martins-Filho, F.

Bellissimo-Rodrigues, C.G. Carlotti Jr. and A. Basile-Filho

The Brazilian Journal of Medical and Biological Research is partially financed by

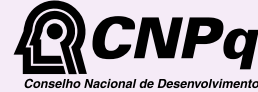

Ministério



da Ciência e Tecnologia

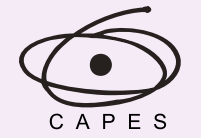

Ministério da Educação

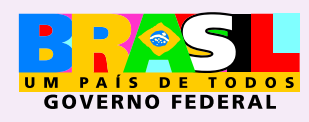

DFAPESP

Institutional Sponsors
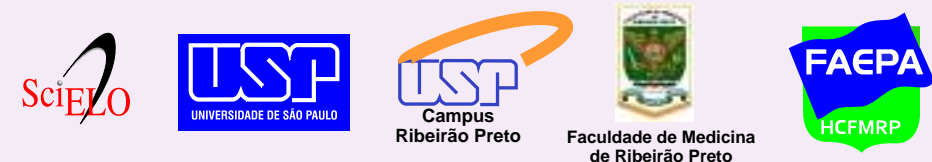

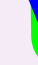

Ф SHIMADZU

GE Healthcare
Hotsite of proteomics metabolomics developped by:

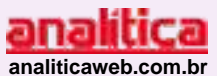

Thermo
sCIEATIFC 


\title{
Meningoencephalitis caused by a zygomycete fungus (Basidiobolus) associated with septic shock in an immunocompetent patient: 1-year follow-up after treatment
}

\author{
M. Auxiliadora-Martins ${ }^{1}$, G.C. Alkmim-Teixeira ${ }^{1}$, J. Machado-Viana1 ${ }^{1}$, E.A. Nicolini ${ }^{1}$, \\ O.A. Martins-Filho ${ }^{4}$, F. Bellissimo-Rodrigues ${ }^{3}$, C.G. Carlotti Jr. ${ }^{2}$ and A. Basile-Filho ${ }^{1}$ \\ ${ }^{1}$ Divisão de Terapia Intensiva, Departamento de Cirurgia e Anatomia, ${ }^{2}$ Divisão de Neurocirurgia, \\ Departamento de Cirurgia e Anatomia, Hospital das Clínicas, ${ }^{3}$ Departamento de Medicina Social, \\ Faculdade de Medicina de Ribeirão Preto, Universidade de São Paulo, Ribeirão Preto, SP, Brasil \\ ${ }^{4}$ Laboratório de Biomarcadores de Diagnóstico e Monitoração, Instituto René Rachou, \\ Fundação Oswaldo Cruz, Belo Horizonte, MG, Brasil
}

\begin{abstract}
Zygomycosis is an infection caused by opportunistic fungi of the Zygomycetes class, specifically those from the Mucorales and Entomophthorales orders. It is an uncommon disease, mainly restricted to immunocompromised patients. We report a case of a 73-year-old male patient with a history of fever $\left(39^{\circ} \mathrm{C}\right)$ lasting for 1 day, accompanied by shivering, trembling, and intense asthenia. The patient was admitted to the intensive care unit with complex partial seizures, and submitted to orotracheal intubation and mechanical ventilation under sedation with midazolam. The electroencephalogram showed evidence of non-convulsive status epilepticus. There is no fast specific laboratory test that permits confirmation of invasive fungal disease. Unless the physician suspects this condition, the disease may progress rapidly while the patient is treated with broad-spectrum antibiotics. Differential diagnosis between fungal and bacterial infection is often difficult. The clinical presentation is sometimes atypical, and etiological investigation is not always successful. In the present case, the histopathological examination of the biopsy obtained from the right temporal lobe indicated the presence of irregular, round, thick-walled fungi forming papillae and elongated structures of irregular diameter, with no septa, indicative of zygomycete (Basidiobolus). Treatment with liposomal amphotericin B and fluconazole was initiated after diagnosis of meningoencephalitis by zygomycete, with a successful outcome.
\end{abstract}

Key words: Zygomycete; Septic shock; Status epilepticus; Meningoencephalitis; ICU setting

\section{Introduction}

Zygomycetes are opportunistic agents that produce infections and cause diseases in situations where the host presents some kind of intrinsic immune disorder. Except for their opportunistic character, little is known about the pathogenesis of zygomycetes, with consequent difficulty in planning treatment, leading to the use of broad-spectrum and highly nephrotoxic antifungal agents, such as amphotericin B. The diseases due to these fungi, which belong to the family Mucoraceae, were initially referred to as mucormycosis. More recently, these diseases have been designated zygomycosis because cases of invasive diseases by fungi belonging to other families or even to the order Entomophthorales have been described (1). In fact, zygomycosis is a broader designation than mucormycosis since it is an infection by opportunistic fungi belonging to the Zygomycetes class, which in turn is divided into two orders: Mucorales and Entomophthorales. These two orders are responsible for different types of severe infections. The genera rhizopus, absidia, and mucor are the most frequent pathological agents within the Zygomycetes class $(1,2)$. Zycomycosis has several types of clinical manifestations including rhinocerebral, gastrointestinal, pulmonary, and

Correspondence: M. Auxiliadora-Martins, Divisão de Terapia Intensiva, Departamento de Cirurgia e Anatomia, Hospital das Clínicas, FMRP, USP, Av. Bandeirantes, 3900, 2º andar, 14049-900 Ribeirão Preto, SP, Brasil. Fax: +55-16-3602-2439.

E-mail: mamartins@hcrp.usp.br/mam_martins@hotmail.com

Received December 29, 2009. Accepted July 5, 2010. Available online July 23, 2010. Published August 13, 2010. 
subcutaneous infections, as well as burn wound colonization with invasion of blood vessels. The rhinocerebral type is the most dramatic, but also the rarest. It usually starts with manifestations in the paranasal sinuses because airborne spores first enter the body via inhalation (3).

Immunocompromising states such as hematological malignancy, bone marrow or solid organ transplant, neutropenia, diabetes mellitus with or without ketoacidosis, treatment with deferoxamine for iron overload, and corticosteroids predispose patients to infection. The occurrence of zygomycosis in immunocompetent hosts is rare and usually related to trauma. Death rates can approach $100 \%$ depending on the patient's underlying disease and the form of zycomycosis $(1,4-6)$. The main risk factors for fungal infections in hospitalized patients are the use of broad-spectrum antibiotics, chemotherapy, previous colonization, parenteral nutrition, surgery, hemodialysis, prolonged therapy with corticosteroids, cancer, intravascular catheter, invasive mechanical ventilation, neutropenia, HIV infection, diabetes mellitus, severe malnutrition, and invasive procedures. Percutaneous skin lesion and spore inhalation are risk factors for non-hospitalized immunocompetent patients. This report describes a case of meningoencephalitis caused by zygomycetes with a clinical histopathological and mycological diagnosis, affecting an immunocompetent patient.

\section{Case report}

A 73-year-old male patient from Ribeirão Preto, São Paulo, Brazil, was admitted to the Infectious Diseases Unit of the University Hospital, Faculty of Medicine of Ribeirão Preto, on March 23,2008 , with a history of fever $\left(39^{\circ} \mathrm{C}\right)$ lasting for 1 day, accompanied by shivering, trembling, and intense asthenia.

Previous history. The patient had been suffering from tonic-clonic seizures since 2003 and was taking phenytoin at the dose of $200 \mathrm{mg} / \mathrm{day}$. The individual had been diagnosed with mitral stenosis, mitral valve prolapse, and systemic arterial hypertension.

Physical examination upon hospital admission revealed a patient in good general health, blood pressure $=170 / 100$ $\mathrm{mmHg}$, heart rate $=80 \mathrm{bpm}$, temperature $=39^{\circ} \mathrm{C}$, and left inferior quadrantopsia. Five days after hospital admission, the consciousness level worsened (Glasgow coma score = 11) and the patient presented asymmetry of muscle force in the left arm as well as recurrent complex partial seizures. Laboratory examinations upon admission to the intensive care unit (ICU) revealed: leukocytes $=13,700 / \mathrm{mm}^{3}$, glycemia $=112 \mathrm{mg} / \mathrm{dL}$, sodium = $136 \mathrm{mEq} / \mathrm{L}$, potassium = $2.8 \mathrm{mEq} / \mathrm{L}$, urea $=46 \mathrm{mg} / \mathrm{dL}$, creatinine $=1.1 \mathrm{mg} / \mathrm{dL}$, cerebrospinal fluid: cells $=40.6$, lymphocytes $(99 \%)$, red blood cells $=53.3$, glucose $=77 \mathrm{mg} / \mathrm{dL}$, and proteins $=87 \mathrm{mg} / \mathrm{dL}$.

Various blood culture samples were collected for fungal search, and results were negative throughout the patient's hospital stay. A search for fungi in the cerebrospinal fluid was performed by counter-immunoelectrophoresis, with negative results for paracoccidioidomycosis, histoplasmosis, and candidiasis.

Magnetic resonance imaging $(\mathrm{MRI})$ of the brain revealed the presence of right fronto-temporal hyperintensity with expansive effects in cortical gyri and extension to the hippocampus. The electroencephalogram (EEG) provided evidence of non-convulsive status epilepticus. Herpes encephalitis was suggested and therapy with $700 \mathrm{mg}$ acyclovir $8 / 8 \mathrm{~h}$ was initiated. The consciousness level of the patient deteriorated, the fever did not cease $\left(38.3^{\circ} \mathrm{C}\right)$, and the patient developed generalized urticariform erythema. The patient was admitted to the ICU on March 31 with complex partial seizures, and submitted to orotracheal intubation and mechanical ventilation under sedation with midazolam. Physical examination revealed an agitated, disoriented, blushing, hydrated, acyanotic, feverish individual with regular general health, respiratory rate $=32$ inspirations per minute, heart rate $=110$ beat per minute, blood pressure $=$ $170 / 110 \mathrm{mmHg}$, presence of a systolic murmur in the mitral focus, and pulmonary base crackles. The APACHE II score (7) was 19 , with a death risk of $21 \%$. On the second day in the ICU, the patient developed oliguria $(510 \mathrm{~mL} / 24 \mathrm{~h})$, urea $=76 \mathrm{mg} / \mathrm{dL}$, creatinine $=4.1 \mathrm{mg} / \mathrm{dL}$. Acute renal failure was detected following the use of acyclovir, with indication for hemodialysis. The patient had fever, presented pulmonary secretion and a chest $\mathrm{X}$-ray revealing opacification in the right pulmonary base compatible with the diagnosis of ventilator-associated pneumonia. On the third day in the ICU, he presented hypotension, which did not improve with volume infusion and norepinephrine was started.

A new MRI examination showed an expansive lesion of the temporal lobe (hypersignal) with apparent invasion of the hypothalamus and the thalamus on the right, and the presence of leptomeningeal enhancement surrounding the temporal lesion. There were no signs of cerebral edema (Figure 1). A direct histopathological examination of the biopsy obtained from the right temporal lobe evidenced the presence of irregular, round, thick-walled fungi forming papillae and elongated structures of irregular diameter, with no septa, indicative of zygomycete (Basidiobolus). Treatment with liposomal amphotericin $B\left(2 \mathrm{mg} \cdot \mathrm{kg}^{-1}\right.$. day $\left.{ }^{-1}\right)$ and fluconazole ( $400 \mathrm{mg} /$ day) was initiated after diagnosis of meningoencephalitis by zygomycete (basidiobolomycosis), with a successful outcome. Administration of liposomal amphotericin B was continued for 21 days, reaching an accumulated dose of $2230 \mathrm{mg}$. After a 24-day stay in the ICU, the patient was discharged to the ward in satisfactory clinical conditions and 9 days later, he was discharged from the hospital.

An EEG carried out at the outpatient clinic in March 2009 revealed findings consistent with a post-inflammation event, with slight disorganization of the right temporal areas. MRI performed on the same date demonstrated areas of encephalomalacia in the right temporal lobe and marginal 


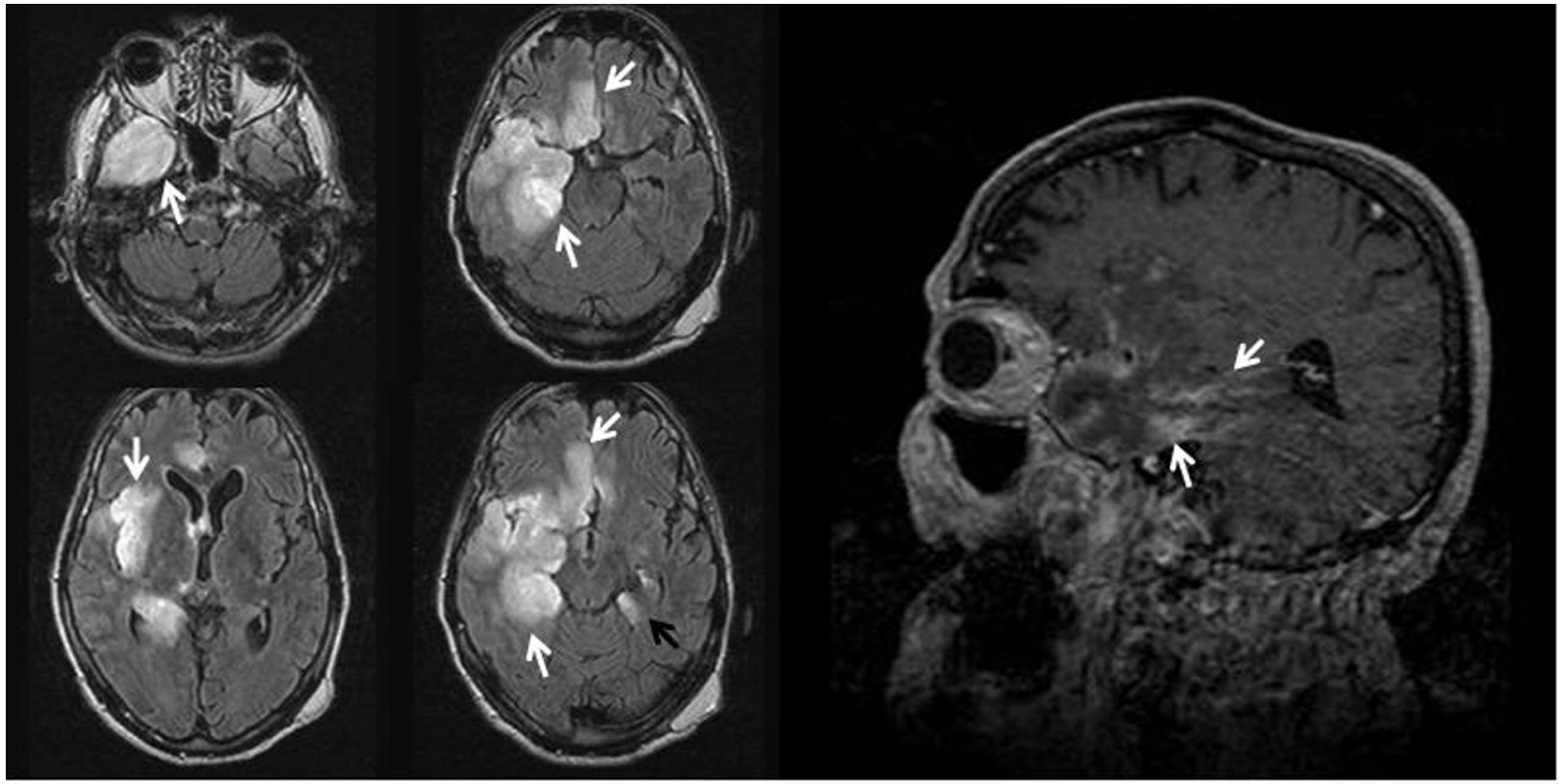

Figure 1. MRI. Lesion showing hypersignal on the fronto-insular-temporal region (white arrows) with apparent contralateral involvement (black arrow), and the presence of leptomeningeal enhancement surrounding the temporal lesion (white arrows) (T1-weighted contrast MRI - bottom right). There were no signs of cerebral edema.

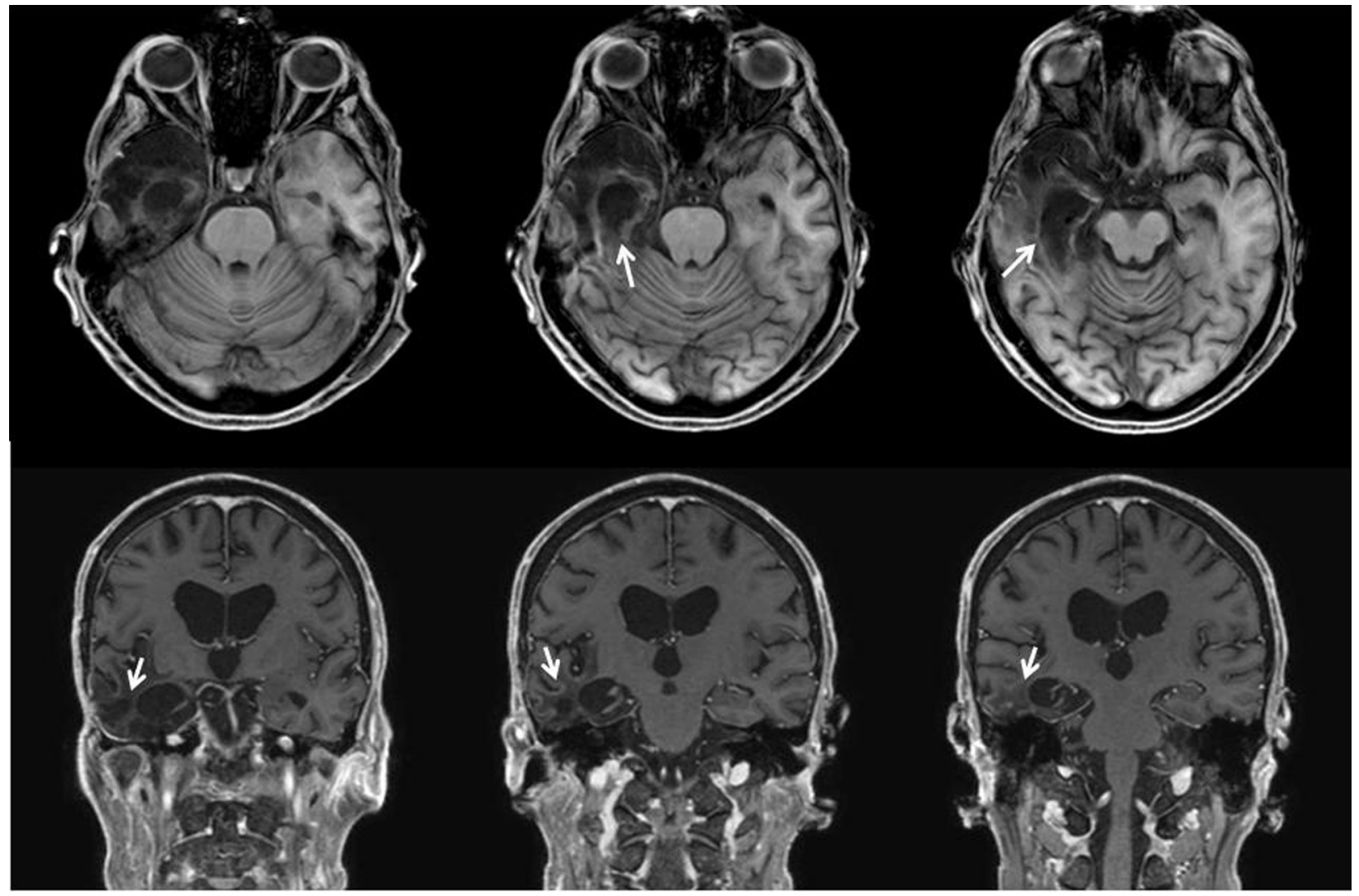

Figure 2. MRI. Focal encephalomalacia in the right temporal lobe (white arrows), without major imaging abnormalities. 
gliosis, without major neurological disorders (Figure 2).

The patient is currently under follow-up and shows good overall clinical conditions. Neurological examination performed in June 2010 revealed normal levels of consciousness. The mini-mental state examination, the most commonly used instrument for screening cognitive impairment, was also carried out and revealed normal cognitive function with a final score of 28 points. The patient was also submitted to the verbal fluency test (animal category) and named 15 animals in $1 \mathrm{~min}$, a result considered normal for his schooling level. This test is a kind of psychological test in which participants have to say as many words as possible from a category in a given time (usually $60 \mathrm{~s}$ ). This category can be semantic, such as animals or fruits, or phonemic, such as words that begin with a given letter.

We also performed the clock-drawing test, which yielded a total score of 10 points. The clock-drawing test is a simple neuropsychometric instrument that can be easily applied to assess several cognitive functions. Many cortical, subcortical, anterior, posterior, right and left skills in brain hemispheres have to operate simultaneously to draw a clock, particularly involving the frontal, temporal and parietal regions. The test displays a sensitivity of $78 \%$ and specificity of $96 \%$ for a score of 10 points. Additionally, we submitted the patient to the evaluation of daily life activities using the modified Barthel Index, a scale commonly used to measure disability or dependence in activities of daily living. The test yielded a total of 100 points, which demonstrated that the patient was considered to be fully independent to perform his daily activities.

\section{Discussion}

Despite the rise in the frequency of zycomycosis over the last 10 years $(8,9)$, there are few reports on this infection in immunocompetent patients. We report here a severe case of zycomycosis caused by fungi of the order Entomophthorales, family Basidiobolaceae and genus Basidiobolus, which resulted in meningoencephalitis and septic shock in an immunocompetent individual.

Usually, opportunistic diseases in the ICU setting occur in patients with comorbidity due to extremely severe illnesses. Diagnosis is slow and hard because of the low efficacy of commercially available tests, poor mycology laboratory infrastructure, and difficult access to some body organs, which in turn hinders biopsy procedures. Death rates are high, and there has been an upsurge in mortality during the last years. The current clinical scenario of fungal infections is thus characterized by increasing incidence, difficult diagnosis, and high mortality. Consequently, antifungal prophylaxis and empirical therapeutics are increasingly being employed. Once they are identified, treatment against these fungi is usually successful (10). Routine tests normally isolate up to zygomycete or to the genus, at most. In the most comprehensive review of zygomycosis published to date, a high percentage of the 929 cases reviewed lacked identification at the species level, and identification was dubious in many of them (11). Over the last few years it has been demonstrated that DNA sequence analysis, especially in the case of ribosomal DNA (rDNA), is useful for the identification of zygomycetes $(12,13)$.

Early diagnosis, together with treatment of the underlying disease, surgery and therapy with amphotericin B are necessary for a successful outcome. Genera of the order Entomophthorales can produce chronic subcutaneous infection in immunocompetent patients. Infection takes place in tropical and subtropical climates. The genus Basidiobolus typically leads to subcutaneous infection of the thigh, buttock, and/or trunk. Involvement of the gastrointestinal tract has rarely been reported. The genus Conidiobolus causes chronic infection of the nasal submucosa and subcutaneous tissue of the nose and face (14). The diagnosis of zygomycosis is based on clinical examination and is confirmed by biopsy, with detection of nonseptate hyphae with right-angle branches and tissue invasion (15).

The hyphae of zygomycetes appear to be thinner-walled compared with other fungal hyphae, a fact that possibly accounts for their weaker staining with Gomori methenamine silver and periodic acid-Schiff relative to fungi with thicker cell walls (16). Culture samples can be used to identify the species, although they may not reveal isolation even in the presence of disseminated and aggressive disease.

There is no fast specific laboratory test that enables confirmation of invasive fungal disease. Unless the physician suspects this condition, the disease may progress rapidly while the patient is treated with broad-spectrum antibiotics. Differential diagnosis between fungal and bacterial infection is often difficult. Presentation is sometimes atypical, and etiological investigation is not always successful. It is important to highlight the role of diagnostic investigation through biopsy by bronchoscopy or thoracoscopy, and/or biopsy of cutaneous-mucosal or brain lesions. The medical staff should not await the final result to initiate fungal therapy. Microscopy examination of the tissues is very important for diagnosis, mainly to detect the presence of nonseptate hyphae, which is an indication of zycomycosis. Culture is usually not very sensitive. In approximately 30 to $40 \%$ of cases, nonseptate hyphae are detected in the samples used for tissue biopsy, but culture is negative. Nevertheless, one approach to searching for this type of fungus is its molecular identification (12,13,17-19). Recently, Alvarez et al. (20) identified 190 strains of zygomycetes that had been previously classified on the basis of morphology. By sequencing the internal transcribed spacer region of the rDNA, these investigators demonstrated that Rhizopus oryzae corresponded to $44.7 \%$ of the fungi identified. In agreement with the present study, the predominant clinical manifestations were the rhinocerebral (25.8\%), pulmonary $(26.8 \%)$, and cutaneous (28\%) forms. Alvarez et al. (20) concluded that identification of zygomycetes still is a hard 
and laborious task, and that morphological features alone may provide a precise diagnosis if assessed by experienced professionals. The case reported here shows that zygomycosis can also affect patients with no history of immunocompromising states, and that therefore this disease should be considered in patients presenting meningoencephalitis of undetermined ethiology.

\section{References}

1. Ribes JA, Vanover-Sams CL, Baker DJ. Zygomycetes in human disease. Clin Microbiol Rev 2000; 13: 236-301.

2. Diwakar A, Dewan RK, Chowdhary A, Randhawa HS, Khanna G, Gaur SN. Zygomycosis - a case report and overview of the disease in India. Mycoses 2007; 50: 247-254.

3. Freifeld AG, Iwen PC. Zygomycosis. Semin Respir Crit Care Med 2004; 25: 221-231.

4. Greenberg RN, Scott LJ, Vaughn HH, Ribes JA. Zygomycosis (mucormycosis): emerging clinical importance and new treatments. Curr Opin Infect Dis 2004; 17: 517-525.

5. Spellberg B, Edwards J Jr, Ibrahim A. Novel perspectives on mucormycosis: pathophysiology, presentation, and management. Clin Microbiol Rev 2005; 18: 556-569.

6. Metellus P, Laghmari M, Fuentes S, Eusebio A, Adetchessi $T$, Ranque $S$, et al. Successful treatment of a giant isolated cerebral mucormycotic (zygomycotic) abscess using endoscopic debridement: case report and therapeutic considerations. Surg Neurol 2008; 69: 510-515.

7. Knaus WA, Draper EA, Wagner DP, Zimmerman JE. APACHE II: a severity of disease classification system. Crit Care Med 1985; 13: 818-829.

8. Brown J. Zygomycosis: an emerging fungal infection. Am J Health Syst Pharm 2005; 62: 2593-2596.

9. Chayakulkeeree M, Ghannoum MA, Perfect JR. Zygomycosis: the re-emerging fungal infection. Eur J Clin Microbiol Infect Dis 2006; 25: 215-229.

10. Rogers TR. Treatment of zygomycosis: current and new options. J Antimicrob Chemother 2008; 61 (Suppl 1): i35-i40.

11. Roden MM, Zaoutis TE, Buchanan WL, Knudsen TA, Sarkisova TA, Schaufele RL, et al. Epidemiology and outcome of zygomycosis: a review of 929 reported cases. Clin Infect Dis 2005; 41: 634-653.

\section{Acknowledgments}

We are thankful to Fundação de Amparo ao Ensino, Pesquisa e Assistência (FAEPA) do Hospital das Clínicas and Faculdade de Medicina de Ribeirão Preto, USP, for financial support.

12. Schwarz P, Bretagne S, Gantier JC, Garcia-Hermoso D, Lortholary O, Dromer F, et al. Molecular identification of zygomycetes from culture and experimentally infected tissues. J Clin Microbiol 2006; 44: 340-349.

13. Voigt K, Wostemeyer J. Phylogeny and origin of 82 zygomycetes from all 54 genera of the Mucorales and Mortierellales based on combined analysis of actin and translation elongation factor EF-1alpha genes. Gene 2001; 270: 113-120.

14. Prabhu RM, Patel R. Mucormycosis and entomophthoramycosis: a review of the clinical manifestations, diagnosis and treatment. Clin Microbiol Infect 2004; 10 (Suppl 1): 31-47.

15. Kontoyiannis DP, Wessel VC, Bodey GP, Rolston KV. Zygomycosis in the 1990s in a tertiary-care cancer center. Clin Infect Dis 2000; 30: 851-856.

16. Frater JL, Hall GS, Procop GW. Histologic features of zygomycosis: emphasis on perineural invasion and fungal morphology. Arch Pathol Lab Med 2001; 125: 375-378.

17. Iwen PC, Freifeld AG, Sigler L, Tarantolo SR. Molecular identification of Rhizomucor pusillus as a cause of sinus-orbital zygomycosis in a patient with acute myelogenous leukemia. J Clin Microbiol 2005; 43: 5819-5821.

18. White MM, James TY, O'Donnell K, Cafaro MJ, Tanabe Y, Sugiyama J. Phylogeny of the Zygomycota based on nuclear ribosomal sequence data. Mycologia 2006; 98: 872-884.

19. Voigt K, Cigelnik E, O'Donnell K. Phylogeny and PCR identification of clinically important Zygomycetes based on nuclear ribosomal-DNA sequence data. J Clin Microbiol 1999; 37: 3957-3964.

20. Alvarez E, Sutton DA, Cano J, Fothergill AW, Stchigel A, Rinaldi MG, et al. Spectrum of zygomycete species identified in clinically significant specimens in the United States. J Clin Microbiol 2009; 47: 1650-1656. 